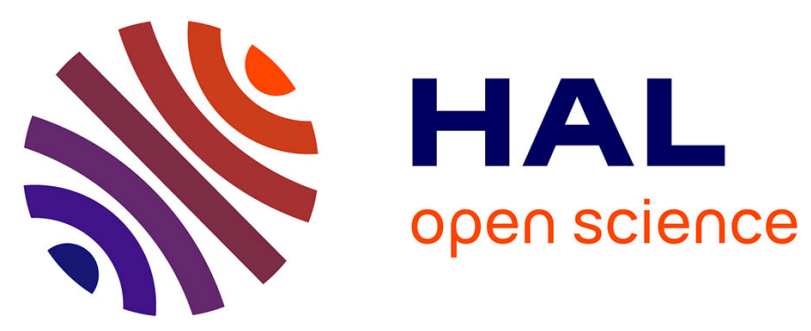

\title{
Precursors and triggering mechanisms of granular avalanches
}

\author{
Renaud Delannay, Mickaël Duranteau, Vincent Tournat
}

\section{To cite this version:}

Renaud Delannay, Mickaël Duranteau, Vincent Tournat. Precursors and triggering mechanisms of granular avalanches. Comptes Rendus. Physique, 2015, 16 (1), pp.45-50. 10.1016/j.crhy.2015.01.002 . hal-01123946

\section{HAL Id: hal-01123946 \\ https://hal.science/hal-01123946}

Submitted on 7 Mar 2015

HAL is a multi-disciplinary open access archive for the deposit and dissemination of scientific research documents, whether they are published or not. The documents may come from teaching and research institutions in France or abroad, or from public or private research centers.
L'archive ouverte pluridisciplinaire HAL, est destinée au dépôt et à la diffusion de documents scientifiques de niveau recherche, publiés ou non, émanant des établissements d'enseignement et de recherche français ou étrangers, des laboratoires publics ou privés. 


\title{
Precursors and triggering mechanisms of granular avalanches Précurseurs et mécanismes de déclenchement d'avalanches granulaires
}

\author{
R. Delannay ${ }^{\mathrm{a}}$, M. Duranteau ${ }^{\mathrm{b}}, \mathrm{V}$. Tournat ${ }^{\mathrm{c}}$ \\ ${ }^{a}$ Institut de Physique de Rennes, UMR CNRS 6251, Université de Rennes 1, Campus de Beaulieu \\ Bâtiment 11A, 263 av. Général Leclerc, 35042 Rennes CEDEX, France \\ ${ }^{b}$ Institut Jean le Rond d'Alembert - UMR CNRS 7190, Sorbonne Universités, UPMC Université Paris \\ 6, 4 place Jussieu, 75005, Paris, France \\ ${ }^{c}$ LAUM, LUNAM Université, CNRS, Université du Maine, Av. O. Messiaen, 72085 Le Mans, France
}

\begin{abstract}
The dynamics of inclined granular packings driven towards their stability limits are studied experimentally using imaging techniques as well as acoustic methods. The formers allow to study grain rearrangements during the tilting. The implementation of both passive and active acoustic methods for probing the granular packings, with capabilities for time resolved measurements, provides information on various elastic properties of the layers along the destabilization process, including the transient precursors. Systematic experiments of granular layer destabilization for various granular media and external conditions are compared and allow to better understand the mechanisms responsible for the appearance, periodicity and intensity of precursors.

La dynamique des empilements granulaires inclinés jusqu'à leur limite de stabilité peut être étudiée à l'aide d'expériences utilisant des techniques d'imagerie ou des méthodes acoustiques. Les premières permettent l'étude des réarrangements de grains pendant l'inclinaison. L'implémentation de méthodes acoustiques passives et actives pour le sondage d'empilements granulaires en temps réel, permet d'estimer la variation des propriétés élastiques au cours du processus de déstabilisation, incluant le régime des précurseurs. Des expériences systématiques de déstabilisation de couches granulaires pour divers milieux granulaires et conditions extérieures sont comparées et permettent d'avoir une meilleure compréhension des mécanismes responsables de l'apparition, de la périodicité et de l'intensité des précurseurs.
\end{abstract}

Keywords: granular media, precursor of avalanche, acoustics

\section{Introduction}

The existence of the unjamming transition plays a large part in the interest of granular materials. The latter are jammed at rest and can sustain some load, but if a threshold

\footnotetext{
*Corresponding author

Email address: renaud.delannay@univ-rennes1.fr (R. Delannay)

Preprint submitted to CR Physique
} 
shear stress is exceeded, part of the material starts to flow. In response to some change in external applied forces, the macroscopic activity of a granular system is related to the evolving geometry of its contact network and to the nature of the contacts. It leads to complex behaviours of great interest for industrial and natural processes. In nature, geological processes like landslides or rock avalanches involve an unjamming transition of granular media. Other natural events like earthquakes are often seen as interacting elements which discharge collectively when they reach a trigger threshold $[1,2]$. The corresponding models are closely related to avalanches models.

For free surface flows of granular systems under the action of gravity, the unjamming transition above a critical shear stress is evidenced by the existence of an angle of maximum stability of a pile: $\theta_{M}$. It is the angle at which the flow starts, the angle of the pile relaxes then towards the smaller angle of repose. Many studies have been devoted to these angles, but must of them focus on the succession of avalanches in a rotating drum $[3,4,5]$, or on a continuously fed pile $[6,7,8]$. In both cases, the heap is built by successive avalanches, giving a specific contact network geometry to the bulk. An alternative method of investigating avalanche dynamics is to incline gently an undisturbed granular bed in the gravity field.

Exploration of the dynamical response of an inclined granular packing before the avalanche starts is of the greatest interest as it allows a study of the dynamical transition from a static packing to a flowing one and brings information which may be helpful for the prediction of the occurrence of the avalanche. Obviously the capability of predicting the probability of occurrence is an important motivation for this area of research.

\section{Avalanche precursors}

Freshly prepared piles filling a box were used to study rearrangements at the surface before the first avalanche $[9,10,11]$. First, small rearrangements implying only a few grains are detected. The size and the rate of these rearrangements increase with the inclination angle. At some stage, large amplitude and quasi periodical events are observed (Fig. 3b). These events, called precursors, consist of collective motions of grains. A correlation between the pseudo period of the precursors and the number of layers gives some clues that these precursors are not only superficial events.

More recent results [12] confirmed that the precursors are bulk phenomena and allowed to interpret these events as reorganizations of the weak-contact sub network occurring in the packing. This is in agreement with computer simulations of inclined 2D packings $[13,14]$ which revealed the occurrence of intermittent rearrangements of grain contacts in the bulk. However beyond the observation of experimental and numerical evidence of the existence of precursors to avalanches, these regular events have never been explained theoretically.

This oscillating behavior is reminiscent of stick-slips originating from difference between static and dynamic frictions in frictional system submitted to an increasing load at a small rate. By the way, seismic processes are sometimes modeled by arrays of sliders connected by springs (Burridge-Knopoff model [15]). Moreover, granular assemblies sheared between planes also exhibit a stick-slip behavior [16] and have been used to model fault gouges. Regular precursors are observed before the onset of frictional motion, and in the mechanical response of amorphous media as metallic glasses and granular materials, before the rupture (see [17] and references herein). More generally, physical systems 
responding through abrupt events to slowly increasing stress are rather abundant, earthquakes have already been held up, plastic bursts in crystals are another example [18]. They often can be seen as the depinning of an interface under an external field. Within the waiting intervals between the fast, abrupt events, a slow restructuring of the pinning field can occure; stresses may be relaxed through competing processes, such smooth responses can have effects on the abrupt events properties. These slow processes may be important for intermittency. As proposed in [18], whenever avalanches compete with slow coexisting processes to minimize the local internal stress, the dynamics give rise to a self-organized avalanche oscillator.

\section{Investigating the behavior of granular avalanche precursors}

Beside the numerical simulations of 2D and 3D [19] inclined granular packings, various experimental methods have been used to expose the secrets of these strange happenings: direct observation of the surface or the side of the box with a camera $[20,21,10]$, X ray imaging technique [22], interferometric technique based on diffusive wave spectroscopy [17], and acoustic methods.

Optical measurements give access to grain motion and allow to measure the precursor pseudo period. Many parameters influence those quantities [21, 17, 19, 23]: the material characteristics of the grains themselves (shape, surface features, size distribution ...), environmental parameters (humidity, vibrations, triggering ...), length, width and height of the packing. Most of these parameters affect the contacts between grains, their capacity to influence the precursors is not surprising. In the same way, the preparation of the packing plays an important role $[22,24,20]$, sometimes neglected by authors who get results seemingly paradoxical. It never should be forgotten that it is the force network which is submitted to strong modifications during the loading. Those modifications are the substance of the precursors, the preludes of the destabilization of the system. In such a context, the acoustic methods of detection, which are directly sensitive to force network restructuring, provide a particularly adapted tool of study.

Acoustic methods can be experimentally used to detect precursors [25, 26, 27] and to probe internal rearrangements in the bulk of the granular layer [12, 24, 28, 27, 29]. Because part of the acoustic wave energy transports through the contact network and the elastic beads of the medium, the probing methods based on the monitoring of acoustic signatures are sensitive to changes in the elastic properties of the granular layer. This class of methods is consequently interesting because it provides sometimes unique, and often complementary, information compared to optical methods [30, 21, 17].

In the following, we give an overview of the acoustic probing methods, mainly implemented by us and our collaborators in the experiments of granular layer destabilization. They can be divided in two groups, the passive methods where acoustic sensors are used to listen to the sounds emitted by the destabilized layer itself and the active methods where an acoustic signal with desired properties is generated and detected by transducers in the medium. The reported results are obtained with $2 \mathrm{~mm}$ diameters monodisperse glass beads assemblies of relatively loose volumic fraction $(0.59-0.60)$. 


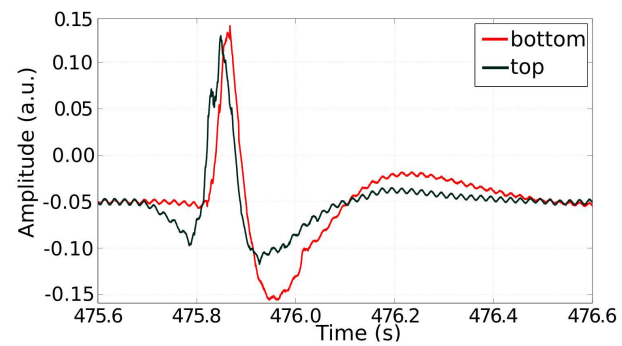

(a)

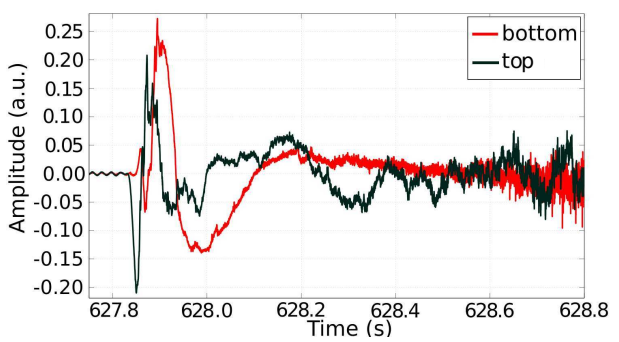

(b)

Figure 1: Typical temporal signal of acoustic emission at a precursor (a) and at an avalanche (b) recorded by two hydrophones buried at half depth in the bulk of the layer. One is placed in the upstream part of the packing when flowing and the second is placed in the downstream part of the packing.

Signaux temporels typiques de l'émission acoustique d'un précurseur (a) et d'une avalanche (b) enregistrés par deux hydrophones enterrés à demi-profondeur dans le volume. L'un est placé à l'amont de l'empilement par rapport au sens de l'écoulement, et le second est situé à l'aval.

\section{Passive acoustic probing}

Passive acoustic probing consists in recording the acoustic emissions originating from the grain and contact local rearrangements (collision, slip motion, ...). After being generated by grain motion, the vibrations propagate via the grain contact network and reach the walls of the vessel. By listening to the sound pulses generated by the medium itself during precursors (Fig. 1a), the latter are easily detected with a time resolution of less than a ms $[25,26,27]$. These acoustic emissions become of higher amplitudes with the angle of inclination and they have relatively strong energy levels compared to the background noise. These emissions recorded inside the packing (Fig. 1) are generally in a long wavelength regime compared to the bead size (frequencies $\sim 10-100 \mathrm{~Hz}$ and wavelengths in the order of $10-100 \mathrm{~cm}$ ). These frequencies and wavelengths seem to be selected by the total size of the packing (resonances of the sample) or by the frequency dependent acoustic attenuation which is in favor to the low frequencies below a cut-off frequency [31].

During the collective motion of grains, acoustic emissions are at first recorded at the top of the packing. Moreover the acoustic emission before the noise of the avalanche (Fig. 1b) is similar to that of a precursor, suggesting that an avalanche is triggered by a precursor occurring at the critical state.

The number of grains mobilized by a precursor increases with the tilting angle (or proximity to the critical event).

It is worth noticing that low frequency sound can be generated on some dunes when sand is sheared, a phenomenon known as the song of dunes [32, 33]. It is striking to see that the related acoustic emissions are also asymmetric, similarly to precursor emissions. Yet, no formal link between these two processes has been established to our knowledge. 


\section{Active acoustic probing}

\subsection{Nonlinear method}

Zaitsev et al. [12, 24] applied a nonlinear acoustic method to probe granular packing destabilizations. This class of methods has been shown to be preferentially sensitive to modifications of the weakest contacts (or weak contact network) in the granular packings $[34,35,36]$, based on considerations using the Hertz-Mindlin theory [37, 38] and a series of different experiments.

One interesting nonlinear probing method uses the self-demodulation effect involved in the parametric antenna operation in underwater acoustics from the 50's [39]. If a modulated sine wave of dynamic strain $\tilde{\epsilon}$ with amplitude $a$ is generated in a nonlinear medium, with a carrier frequency $\omega$ and an amplitude modulation frequency $\Omega$, then this signal is demodulated and a new frequency component $\Omega$ is generated in the medium (Fig. 2a).

$$
\tilde{\epsilon}=a[1+\cos (\Omega t)] \cos (\omega t),
$$

Considering heuristically strong (with static strain $\epsilon_{0}$ larger than the average) and weak contacts (with much smaller static strains $\mu \epsilon_{0}$ with $\mu \ll 1$ ) for describing the bead contact network, with the proportions $A$ and $B$ respectively for each family, the acoustic (dynamic) stresses $\tilde{\sigma}_{\omega}, \tilde{\sigma}_{2 \omega}$ and $\tilde{\sigma}_{\Omega}$ can be approximated as the following, in the frame of the Hertz theory of contacts $[12,36]$ :

$$
\tilde{\sigma}_{\omega} \propto A \sqrt{\epsilon_{0}}\left(1+\frac{B \sqrt{\mu}}{A}\right) \tilde{\epsilon}, \tilde{\sigma}_{2 \omega, \Omega} \propto \frac{A}{\sqrt{\epsilon_{0}}}\left(1+\frac{B}{A \sqrt{\mu}}\right) \tilde{\epsilon}^{2} .
$$

The nonlinear component at $\Omega$ is preferentially sensitive to modifications of the weakest contacts because $\mu^{-\frac{1}{2}}$ is large. The authors respectively choose $\omega$ and $\Omega$ around 10 and $1 \mathrm{kHz}$ that is nearly 1 and 10 bead diameters respectively. They probe the packing with very large transducers compare to the size beads in a noninvasive way to avoid clapping contacts $\left(\tilde{\epsilon} \ll \epsilon_{0}\right)$. In this case, strong correlations between surface and bulk rearrangements can be revealed (Fig. 3) [27, 28]. They show strong variations of the weak contact network during the tilt especially just before avalanche triggering (Fig. 2b). However this technique does not allow to localize the internal rearrangements because acoustic waves affect almost all the bulk. By contrast with the nonlinear component, the linear one remains stable during the tilt, a behaviour due to its propagation regime which depends of the frequency (see Sec. 5.2). These acoustic results are compared with surface measurements made simultaneously and the authors conclude that correlations between optical and acoustical signals seem difficult to establish. However a quasi-periodic trend is discernable for the last degrees of inclination for the amplitude of the nonlinear component at $\Omega$ in the Fourier domain. They conclude that internal rearrangements of the weakest contacts are linked to precursors.

Furthermore, this nonlinear acoustic method was used to probe aging effects when a series of consecutive forth-and-back tilting cycles, with an inclination angle varying between 0 and a maximum value smaller than $\theta_{M}$, is made $[24,40]$. The acoustic signature variations demonstrate the anisotropy of the contact orientations in the network of weak contacts [41]. Mirror-type precursors are detected in the bulk with weaker and weaker amplitude variations after several forth-and-back tilting cycles. After several consecutive tilts of a freshly prepared packing, the rearrangements become hardly visible. 


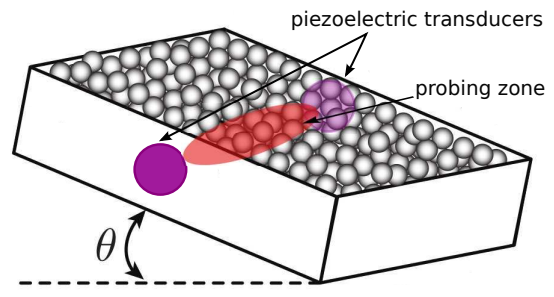

(a)

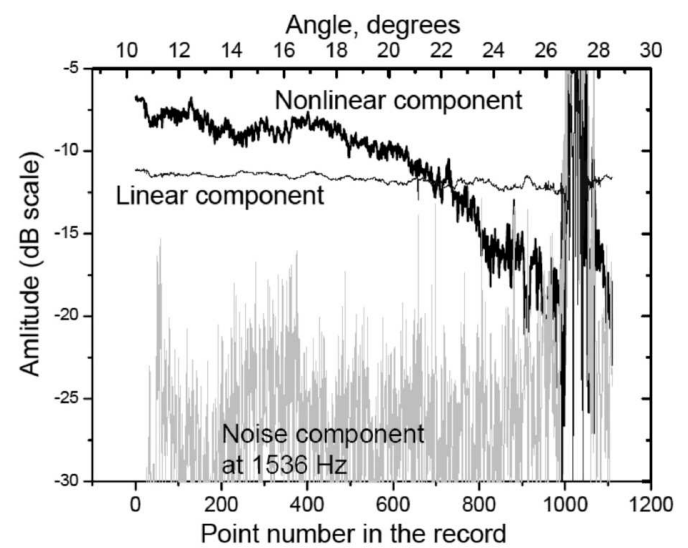

(b)

Figure 2: Experiment of Zaitsev et al. [12] where a tilted packing is probed by acoustic waves. (a) Two piezoelectric transducers are placed in each lateral wall in contact with grains. (b) Linear $\omega$ and nonlinear $\Omega$ component evolutions until avalanche.

Expériences de Zaitsev et al. [12] où un empilement incliné est sondé par des ondes acoustiques. (a) Deux transducteurs piézoélectriques sont placés dans chaque paroi latérale en contact avec les grains. (b) Évolution des composantes fréquentielles linéaire $\omega$ et non linéaire $\Omega$ jusqu’à avalanche.

In a recent study, the experimental setup of acoustic and optical probing of the destabilization of granular layers has been improved with a design and elements resulting in a weaker mechanical noise on the medium. In this case, strong correlations between surface and bulk rearrangements can be revealed (Fig. 3) [27, 28]. The surface rearrangements (Fig. 3b) are measured by the way of a particle tracking method [24] in order to access to the rearranged surface ratio $S / S_{0}$ (with $S_{0}$ the total free surface) and its activity $A$ (the cumulative $S / S_{0}$ with the tilting angle $\theta$ ). The acoustic signal amplitude (Fig. 3a) strongly falls down when a precursor occurs at the surface and this amplitude variation tends to increase at the approach of the avalanche suggesting that more and more contacts break or are modified. In addition, relaxation phenomenon is seen after each precursor assumed to be related to a progressive rebuilding of the contacts which have broken, that is hardening $[23,27]$.

\subsection{Linear method}

The active probing works as well with more conventional linear acoustic methods, in the configuration of relatively loose and confined packings of millimeters monodisperse glass beads. In such configurations, even the strongest contacts are sufficiently weak to be strongly influenced (modified, brought to shear sliding condition or broken) by the tilting process and the small rearrangements. In fact the choice of the working frequency is crucial because acoustic probing at sufficiently low frequencies is sensitive to the solid skeleton formed by the bead contact network [31]. Up to a cut-off frequency which depends mainly on the bead size for gravity stressed granular media, the acoustic propagation is very sensitive to modifications of the average elasticity of the medium. 

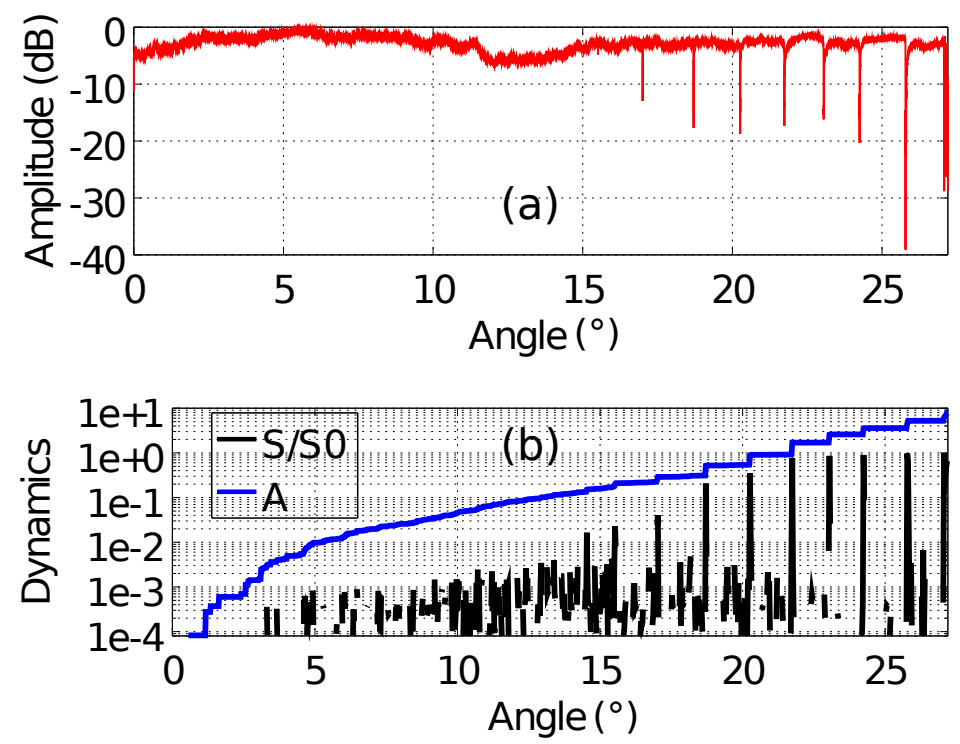

Figure 3: (a) Level of the demodulated component during a tilt of a packing. (b) Dynamics of surface rearrangements.

(a) Niveau de la composante fréquentielle démodulée au cours de l'inclinaison d'un empilement. (b) Dynamique des réarrangments de surface.

Above this cut-off frequency, the detected acoustic signal has mostly propagated through the saturating air and is consequently insensitive to solid elasticity modifications. The measurement of the power spectrum density measured along time or equivalently the angle of inclination below the cut-off frequency shows a shift towards lower frequencies [27]. This can be interpreted as an elastic softening of the medium during a tilt until avalanche: the medium losses progressively its mechanical rigidity. Further measurements of the sound speed and of the central frequency of transmitted pulses indicate the same softening phenomenon as well. Moreover a strong softening effect occurs at each precursor and is followed by a slower relaxation corresponding to hardening. That can be seen as the decrease of a mean number and average rigidity of contacts for softening and the opposite for hardening.

\section{Conclusions and prospects}

To conclude, acoustic methods are powerful tools for the understanding of the destabilization leading to the critical state. Sensitivity to the packing elasticity variations with a ms time resolution can be achieved. Low frequency linear propagation allows to probe the modifications of average elasticity of the medium. Acoustic measurements give direct information on the bulk mobilization, but the measurement volume is relatively large compared to one grain, and the effort required for interpretation can be important. It is thus necessary to combine them with more local methods of characterizing. We generally associate them with optical methods but a combination with numerical simulations is also very promising. 


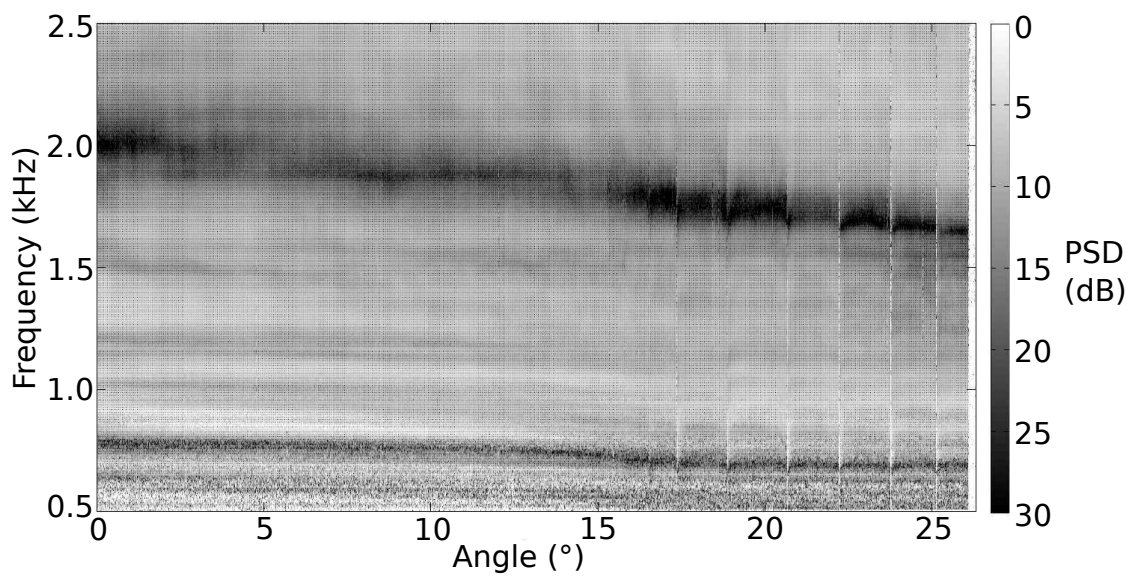

Figure 4: Evolution of the power spectrum density $(P S D)$ measured during a tilt until avalanche. The shift of the spectrum toward lower frequencies indicates softening of the medium.

Évolution de la densité spectrale de puissance (PSD) mesurée durant une inclinaison jusqu'à avalanche. Le décalage du spectre vers les plus basses fréquences indique le ramollissement du milieu.

We thank our collaborators: V.Y. Zaitsev, Y. Le Gonidec, G. Le Caër, A. Faisant and P. Richard. We are also gratefull to V. Gibiat for the review of this present paper.

\section{References}

[1] Z. Olami, J. Feder, K. Christensen, Self-organized criticality in a continuous, nonconservative cellular automaton modeling earthquakes, Physical Review Letters 68 (8) (1992) 1244-1247.

[2] P. Johnson, H. Savage, M. Knuth, J. Gomberg, C. Marone, Effects of acoustic waves on stick-slip in granular media and implications for earthquakes, Nature 451 (3) (2008) 57-61.

[3] R. Fischer, P. Gondret, B. Perrin, M. Rabaud, Dynamics of dry granular avalanches, Physical Review E 78 (2008) 021302.

[4] R. Fischer, P. Gondret, M. Rabaud, Transition by intermittency in granular matter: From discontinuous avalanches to continuous flow, Physical Review Letters 103 (2009) 128002.

[5] GDR-MiDi, On dense granular flows, Eur. Phys. J. E 14 (4) (2004) 341-365.

[6] V. Frette, K. Christensen, A. M. The-Sorenssen, J. Feder, T. Jossang, P. Meakin, Avalanche dynamics in a pile of rice, Nature 379 (1996) 49-52.

[7] H. Jaeger, C. Liu, S. Nagel, Relaxation at the angle of repose, Physical Review Letters 62 (1) (1989) 40-43.

[8] D. V. Denisov, Y. Y. Villanueava, K. A. Lörincz, S. May, R. J. Wijngaarden, Relation between self-organized criticality and grain aspect ratio in granular piles, Physcical Review E 85 (2012) 051309 .

[9] N. Nerone, M. Aguirre, A. Calvo, I. Ippolito, D. Bideau, Surface fluctuations in a slowly driven granular system, Physica A: Statistical Mechanics and its Applications 283 (1-2) (2000) 218 - 222.

[10] N. Nerone, M. Aguirre, A. Calvo, D. Bideau, I. Ippolito, Instabilities in slowly driven granular packing, Physical Review E 67 (2003) 011302.

[11] N. Nerone, S. Gabbanelli, Surface flutuations and the inertia effect in sandpiles, Granular Matter 3 (2001) 117-120.

[12] V. Zaitsev, P. Richard, R. Delannay, V. Tournat, V. Gusev, Pre-avalanche structural rearrangements in the bulk of granular medium: experimental evidence, Europhysics Letters 83 (2008) 64003. 
[13] L. Staron, F. Radjai, J. Vilotte, Granular micro-structure and avalanche precursors, Journal of Statistical Mechanics: Theory and Experiment 7 (2006) 07014.

[14] L. Staron, J. Vilotte, F. Radjai, Preavalanche instabilities in a granular pile, Physical Review Letters 89 (20) (2002) 204302.

[15] Model and theoretical seismicity.

[16] S. Nasuno, A. Kudrolli, J. Gollub, Friction in granular layers: hysteresis and precursors, Physical Review Letters 79 (1997) 949-952. doi:10.1103/PhysRevLett.79.949. URL http://link.aps.org/doi/10.1103/PhysRevLett.79.949

[17] A. Amon, R. Bertoni, J. Crassous, Experimental investigation of plastic deformations before granular avalanche, Physical Review E 87 (2013) 012204.

[18] S. Papanikolaou, D. Dimiduk, W. Choi, J. Sethna, M. Uchic, C. Woodward, S. Zapperi, Quasiperiodic events in crystal plasticity and the self-organized avalanche oscillator, Nature 490 (2012) $517-522$.

[19] L. Oger, A. Vidales, R. Uñac, I. Ippolito, Tilting process with humidity: DEM modeling and comparison with experiments, Granular Matter 15 (2013) 629-643.

[20] N. Gravish, D. Goldman, Effect of volume fraction on granular avalanche dynamics, Physical Review E (2014) 032202.

[21] S. Kiesgen De Richter, G. Le Caër, R. Delannay, Dynamics of rearrangements during inclination of granular packings: the avalanche precursor regime, Journal of Statistical Mechanics: Theory and Experiment (2012) 04013.

[22] A. Kabla, G. Debrégeas, J. Di Meglio, T. Senden, X-ray observation of micro-failures in granular piles approaching an avalanche, Europhysics Letters 71 (6) (2005) 932-937.

[23] M. Duranteau, R. Delannay, P. Richard, V. Tournat, Avalanches and quasi-periodic events in slowly tilted granular media, in preparation.

[24] S. Kiesgen de Richter, V. Zaitsev, P. Richard, R. Delannay, G. L. Caër, V. Tournat, Experimental evidence of ageing and slow restoration of the weak-contact configuration in tilted 3d granular packings, Journal of Statistical Mechanics: Theory and Experiment 11 (2010) 11023.

[25] V. Gibiat, E. Plaza, P. D. Guibert, Acoustic emission before avalanches in granular media, Journal of the Acoustical Society of America 123 (5).

[26] J. Thirot, Y. Le Gonidec, B. Kergosien, Acoustic emissions in multiscale granular structures under gravitational destabilization, in: International Congress of Ultrasonics Proceedings, 2012.

[27] M. Duranteau, V. Tournat, V. Zaitsev, Y. L. Gonidec, P. Richard, R. Delannay, Transient elastic softening/hardening for avalanche precursors of destabilized granular layers probed with acoustic waves, in preparation.

[28] M. Duranteau, V. Tournat, V. Zaitsev, R. Delannay, P. Richard, Identification of avalanche precursors by acoustic probing in the bulk of tilted granular layers, Proceedings of the 7th International Conference on Micromechanics of Granular Media Sydney, Australia (2013) 650-653.

[29] P. Johnson, B. Ferdowsi, B. Kaproth, M. Scuderi, M. Griffa, J. Carmeliet, R. Guyer, D. Le Bas, P. Trugman, C. Marone, Acceleration of acoustical emission precursors preceding failure in sheared granular material, Geophysical Research Letters 21 (40) (2013) 5627-5631.

[30] C. Aegerter, K. Lörincz, M. Welling, R. Wijngaarden, Extremal dynamics and the approach to the critical state: experiments on a three dimensional pile of rice, Physical Review Letters 92 (5) (2004) 058702 .

[31] J.-B. Legland, V. Tournat, O. Dazel, A. Novak, V. Gusev, Linear and nonlinear biot waves in a noncohesive granular medium slab: transfer function, self-action, second harmonic generation, Journal of the Acoustical Society of America 131 (6) (2012) 4292-4303.

[32] S. Douady, A. Manning, P. Hersen, H. Elbelrhiti, S. Protière, A. Daerr, B. Kabbachi, Song of the dunes as a self-synchronized instrument, Physical Review Letters 97 (2006) 018002.

[33] B. Andreotti, The song of dunes as a wave-particle mode locking, Physical Review Letters 93 (2004) 238001.

[34] V. Zaitsev, A. Kolbakov, V. Nazarov, Detection of acoustic pulses in river sand: Theory, Acoustical Physics 45 (3) (1999) 347-353.

[35] V. Zaitsev, A. Kolbakov, V. Nazarov, Detection of acoustic pulses in river sand: Experiment, Acoustical Physics 45 (2) (1999) 235-241.

[36] V. Tournat, V. Zaitsev, V. Gusev, V. Nazarov, P. Béquin, B. Castagnède, Probing weak forces in granular media through nonlinear dynamic dilatancy: clapping contacts and polarization anisotropy, Physical Review Letters 92 (8) (2004) 085502

[37] K. L. Johnson, Contact mechanics, Cambridge University Press, 1985.

[38] L. D. Landau, E. M. Lifshitz, Thoery of elasticity, Pergamon Press, Oxford, 1986. 
[39] B. Novikov, O. Rudenko, V. Timochenko, Nonlinear underwater acoustics, ASA, New York, 1987.

[40] M. Duranteau, Dynamique granulaire à l'approche de l'état critique, Master's thesis, Université de Rennes 1 (2013).

[41] S. Deboeuf, O. Dauchot, L. Staron, J. Vilotte, A. Mangeney, Déformations d'un empilement granulaire lors des cycles de rotation quasi-statique sous gravité, in: 17ème Congrès Français de Mécanique, 2005. 